\title{
Low back pain risk factors associated with persistence, recurrence and delayed presentation
}

\author{
Markus Melloh $^{\mathrm{a}, *}$, Achim Elfering ${ }^{\mathrm{b}}$, Tasha R. Stanton ${ }^{\mathrm{c}, \mathrm{d}}$, Thomas Barz ${ }^{\mathrm{e}}$, Emin Aghayev $^{\mathrm{f}}$, \\ Christoph Röder ${ }^{\mathrm{f}}$ and Jean-Claude Theis ${ }^{\mathrm{g}}$ \\ ${ }^{a}$ Centre for Medical Research, University of Western Australia, Nedlands, WA, Australia \\ ${ }^{\mathrm{b}}$ Department of Work and Organizational Psychology, Institute for Psychology, University of Berne, Berne, \\ Switzerland \\ ' Sansom Institute for Health Research, University of South Australia, Adelaide, SA, Australia \\ ${ }^{\mathrm{d}}$ Neuroscience Research Australia, Sydney, NSW, Australia \\ e Department of Orthopaedic Surgery, Asklepios Klinikum Uckermark, Schwedt/Oder, Germany \\ ${ }_{\mathrm{f}}^{\mathrm{f}}$ Institute for Evaluative Research in Orthopaedic Surgery, MEM Research Center, University of Berne, Berne, \\ Switzerland \\ ${ }^{g}$ Centre for Musculoskeletal Outcomes Research, Dunedin School of Medicine, University of Otago, Dunedin, New \\ Zealand
}

\begin{abstract}
.
BACKGROUND: There is little knowledge in the literature on the role of time-related variables for the prognosis of acute and subacute low back pain (LBP).

OBJECTIVE: The aim of this study was to estimate the relationship between time-related LBP characteristics and prognostic factors for acute/subacute LBP.

METHODS: We performed a prospective inception cohort study of 315 patients attending a health practitioner for acute/subacute LBP or recurrent LBP. One-tailed correlations were conducted between patient characteristics and time-related variables.

RESULTS: The pattern of correlation between risk factors for and resources against persistent LBP differed between three time-related variables. 'Subacute LBP' and 'delayed presentation' were positively associated with psychological factors. Both indicators were negatively correlated with resources against development of persistent LBP. Moreover, 'delayed presentation' was related positively with occupational stressors. In contrast, 'recurrent LBP' was only related to more impaired health-related factors.

CONCLUSIONS: Patients with current LBP waiting longer until seeking help in primary care have a more disadvantageous profile of occupational and psychological risk factors and lower resource levels. A similar but less pronounced pattern occurred in those with subacute LBP compared to those with acute LBP. Consideration of time characteristics of LBP may help to better understand LBP.
\end{abstract}

Keywords: Back pain, prognosis, risk factors, resources, occupational, inception cohort

${ }^{*}$ Corresponding author: Markus Melloh, Centre for Medical Research, University of Western Australia, B Block, QEII Medical Centre, Hospital Avenue, Nedlands, WA, Australia. Tel.: +61 89346 3464; Fax: +61 89346 1818; E-mail: markus.melloh@uwa.edu.au.

\section{Introduction}

It is beyond dispute that early identification of primary care patients with acute $(<$ six weeks) and subacute (six to twelve weeks) low back pain (LBP) at risk of developing persistent LBP is essential $[1,2]$ to reduce the socioeconomic costs and societal burden of LBP [3]. In this patient group, a recent study demon- 
strated that psychological baseline predictor-variables could detect patients at risk of developing persistent LBP up to six months [4]. Screening for persistent pain includes time-related pain characteristics; however, baseline-predictors in screening rarely include time-related LBP characteristics. In sum, there is little knowledge in the literature on the role of timerelated variables, such as waiting until LBP is in the subacute stage before seeking treatment, for the prognosis of acute and subacute LBP [5]. However, subgroup analysis of LBP populations by time-related binary variables such as 'acute/subacute LBP' and 'nonrecurrent/recurrent LBP' or by time-related continuous variables such as 'delayed presentation' (i.e. the time between LBP onset and first health practitioner visit in workdays) may contribute to an understanding of the development of persistent LBP. A first step is to learn more about the association of time-related characteristics of LBP and known risk factors for the development of persistent LBP such as reduced physical activity, as well as resource factors preventing the development of persistent LBP such as high job satisfaction; these factors could be based on the recommendations of the Multinational Musculoskeletal Inception Cohort Study (MMICS) Statement [6]. This study concentrates on three time-related characteristics that may be relevant in inception cohort studies.

Inception cohorts are considered to be the gold standard for cohort studies [7]. They are defined as "consecutive patients identified at the first visit for a problem" [8], or at an "early, uniform point in the course of the specified health condition" [9]. Patients are included in LBP inception cohorts as having acute or subacute LBP based on the time point at which they seek care from their health practitioner for the first time with a new episode of LBP. Delayed care-seeking behaviour in patients with subacute LBP can be due to higher workloads, work stress or the expectation of self-healing.

At the first visit to a health practitioner for patients with acute or subacute LBP, a new episode of LBP is defined as at least 30 LBP-free days before the current episode [10]. A strict definition of an inception cohort is including only patients with non-recurrent episodes [10,11]. A less strict definition is including only patients with recurrent episodes. This variation may result in censorship bias [7].

For patients with a new episode of acute or subacute LBP, longer delay before initial presentation to a health care practitioner may increase the risk of developing persistent LBP. Problem-oriented coping with LBP in- creases self-efficacy in individuals and decreases feelings of helplessness [12]. Patients with severe LBP who do not consult their health care practitioner or aggravate their symptoms, experience fear and worry, including disturbed sleep and reduced resources [13]. Thus, patients may have fewer resources for preventing persistent LBP. Therefore, it is imperative to evaluate the relationship between differences in presentation (e.g. presenting with acute vs. subacute LBP or recurrent vs. non-recurrent LBP or 'delayed presentation') and potential risk factors for persistent LBP. These differences in presentation deemed to be most important were presenting with: 1) a status of subacute LBP at time of first visit to a health practitioner ('subacute LBP'); 2) a history of recurrent LBP ('recurrent LBP'); and 3) a 'delayed presentation' (number of workdays from onset of LBP until first visit to a health practitioner). It would also be informative to determine the relationship between these differences in presentation (i.e. time-related variables) and the presence of resources to prevent persistent LBP. This allows an interesting comparison to see if it is predominantly potential risk factors for persistent LBP that relate to time-related variables or whether it is a lack of resources to prevent persistent LBP.

We hypothesised that

(1) Risk factors for persistent LBP would be positively correlated with the three time-related variables a) 'subacute LBP', b) 'recurrent LBP' and c) 'delayed presentation';

(2) Resources for preventing persistent LBP would be negatively correlated with these time-related variables.

\section{Material and methods}

This study was conducted according to the recommendations of the Declaration of Helsinki (2008) and has been approved by the local Lower South Regional Ethics Committee (LRS/08/03/008). The protocol for the study has been published previously [14].

An inception cohort of 315 consecutive patients was recruited from primary care settings across New Zealand. Patients were invited to participate when attending a health practitioner for their first episode of acute/subacute LBP, or for recurrent LBP as defined by Stanton et al. as LBP with at least 30 LBP-free days between episodes and additionally, exceeding 20 out of 100 points on the Visual Analogue Scale (VAS) [15]. 
Table 1

Time-related LBP characteristics and risk factors for and resources against LBP

\begin{tabular}{|c|c|c|}
\hline & Variables & Description (where appropriate) \\
\hline \multicolumn{3}{|c|}{ Time-related LBP characteristics } \\
\hline Subacute LBP & Acute (0) vs. subacute (1) & New episode of LBP with a duration of six to twelve weeks [1] \\
\hline Recurrent LBP & Non-recurrent (0) vs. recurrent (1) & $\begin{array}{l}\text { At least } 30 \text { LBP-free days between episodes and additionally, ex- } \\
\text { ceeding } 20 \text { out of } 100 \text { points on the Visual Analogue Scale [15] }\end{array}$ \\
\hline Delayed presentation & Number of workdays & $\begin{array}{l}\text { Number of workdays from onset of LBP until first visit to a } \\
\text { health practitioner }\end{array}$ \\
\hline \multicolumn{3}{|r|}{$\mathrm{C}_{\mathrm{P}}$} \\
\hline Lifestyle factors & Smoking (pack/years) & Packs of cigarettes smoked per year \\
\hline Functional limitation & ODI & Oswestry Disability Index \\
\hline \multirow[t]{5}{*}{ Pain } & Sensory pain & All items taken from McGill Pain Questionnaire \\
\hline & Affective pain & \\
\hline & Total pain & \\
\hline & Pain intensity last week & On Visual Analogue Scale (VAS) \\
\hline & Present pain intensity & \\
\hline \multirow[t]{11}{*}{ Psychological factors } & Depression & Defined by ZUNG Depression Index \\
\hline & Somatization & Defined by Modified Somatic Perception Questionnaire \\
\hline & Fear avoidance beliefs & Defined by Fear Avoidance Beliefs Questionnaire \\
\hline & Work activity & \\
\hline & Physical activity & \\
\hline & Pain catastrophizing & Defined by Pain Catastrophizing Score (PCS) \\
\hline & Rumination & To turn a matter over and over in the mind \\
\hline & Magnification & Magnifying one's actual symptoms \\
\hline & Helplessness & \\
\hline & PCS total & \\
\hline & Catastrophizers & Score on PCS of $>24$ out 52 points \\
\hline \multirow[t]{9}{*}{ Occupational factors } & Resigned attitude towards job & $\begin{array}{l}\text { Working in current position due to perceived lack of alternatives, } \\
\text { lowering of job expectations }\end{array}$ \\
\hline & Job content & \\
\hline & Uncertainty & Tasks and goals are not clear \\
\hline & Organisational problems & Equipment and procedures are performance restraints \\
\hline & Work interruptions & \\
\hline & Concentration requirements & \\
\hline & Time pressure & \\
\hline & Unfavourable ergonomics & Prolonged sitting, standing or forward-bending posture \\
\hline & Emotional dissonance & Having to express an emotion one does not feel \\
\hline \multirow[t]{2}{*}{ Expectations } & Expectations going back to work & \\
\hline & Expect. back to work at 6 months & \\
\hline Demographics & Age & \\
\hline \multicolumn{3}{|l|}{ Resources } \\
\hline Lifestyle factors & Physical activity & \\
\hline Education status & Higher education & \\
\hline \multirow[t]{2}{*}{ General health } & SF 12 physical score & Short Form-12 Questionnaire \\
\hline & SF 12 mental score & \\
\hline \multirow[t]{7}{*}{ Occupational factors } & Job satisfaction & \\
\hline & Job content & \\
\hline & Method control & E.g., independently plan and organize one's own work \\
\hline & Time control & E.g., influence on work scale and schedule \\
\hline & Social support & \\
\hline & At work & $\begin{array}{l}\text { Perceived support with problems at work from supervisor and } \\
\text { colleagues }\end{array}$ \\
\hline & At home & $\begin{array}{l}\text { Perceived support with problems at work from friends, spouses } \\
\text { and relatives }\end{array}$ \\
\hline
\end{tabular}

We included patients between 18 and 65 years of age; they had to be able to read and write in English, and to provide written consent. Potential participants were excluded if they had chronic LBP (LBP continuing for more than twelve weeks at the time of the first visit to a health practitioner) [16,17], specific LBP (e.g. infection, tumour, osteoporosis, ankylosing spondylitis, fracture, deformity, inflammatory process, cauda equina syndrome) [18], a comorbidity such as painful disabling osteoarthritis of hip or knee joints compromising overall well-being, or if they no longer had LBP at the time of the screening interview. Additionally, pa- 
tients were excluded if they were currently pregnant or were unable to complete questionnaires.

Patients were screened using a structured standardised phone interview. Eligible patients were sent a questionnaire by mail and requested to return it in a stamped addressed envelope. If not returned, reminders were sent out after one and two weeks. As compensation for their time participants received a \$NZ10 grocery, fuel or book voucher of their choice. The questionnaire was based on the recommendations of the MMICS Statement [6] looking at occupational, psychological, social and biomedical (e.g. pain, disability and physical activity) risk factors for the development of persistent LBP and resources (job satisfaction, method control at work, time control at work, social support at work and at home) for the prevention of persistent LBP (Table 1).

To test Hypothesis 1 and Hypothesis 2, we conducted one-tailed Spearman rank correlations between patient characteristics, (i.e. demographic characteristics, potential risk factors for the development of persistent LBP and resources for the prevention of persistent LBP), and the three time-related variables 'subacute LBP', 'recurrent LBP' and 'delayed presentation'. In addition, in order to confirm significant associations with non-parametrical statistics, acute vs. subacute LBP and non-recurrent vs. recurrent LBP as dichotomous variables and 'delayed presentation' as a continuous variable (number of workdays) were tested using bi-serial Spearman rank correlations and Spearman rank correlation. Descriptive statistics are presented as means \pm standard deviations. Data was analysed using IBM SPSS Statistics 19 (IBM Corp., Armonk, NY, USA). Statistical significance was set at the $P<0.05$ level, one-tailed.

\section{Results}

A total of 562 consecutive patients with acute or subacute LBP were screened between April 2008 and October 2010. One-hundred-and-twenty-four potential participants were ineligible because they were either LBP-free at the time of the screening interview (ten); had been suffering from chronic LBP for more than twelve weeks (93) or from specific LBP (eight); had osteoarthritis of hips and/or knees (two); were pregnant (three); unable to attend assessment (two) or were older than 65 years (six). Twenty-six patients declined to participate and a further 97 did not respond despite two reminders. This resulted in 315 patients who participated in the study.
Two-hundred-and-twenty-two out of these 315 respondents had a first episode of LBP. Ninety-two were classified as having recurrent LBP. One participant did not disclose details of his new LBP episode. Baseline characteristics of the included participants differed from census data of the New Zealand population with regard to a higher education status, lower full-time employment rate, higher percentage of students and professionals, lower percentage of participants working in service or sales, lower age and higher percentage of females in the current study sample [19-21].

Results show mainly positive correlations between time-related LBP characteristics and risk factors, and negative correlations between time-related LBP characteristics and resources (Table 2). The pattern of significant correlations, however, differs between timerelated LBP characteristics. Correlation analyses revealed that psychological and, restricted to 'delayed presentation', occupational risk factors for persistent LBP were significantly correlated with 'subacute LBP' and 'delayed presentation' (Table 3). Resources for preventing persistent LBP were inversely correlated with 'subacute LBP' and 'delayed presentation'. There were fewer significant correlations with 'recurrent LBP' that were restricted to LBP intensity and indicators of general physical health.

'Subacute LBP' demonstrated statistically significant correlations with six prognostic factors; positive correlations with one biomedical factor ('pain intensity last week') and three psychological risk factors ('pain catastrophizing', 'magnification', 'helplessness') and negative correlations with two occupational resources ('job satisfaction', 'time control'). The strongest risk factor was 'magnification', the strongest resource 'time control' (Table 3).

'Recurrent LBP' correlated significantly with seven prognostic factors. Positive correlations with biomedical risk indicators included, 'sensory', 'affective' and 'total pain' as well as 'pain intensity in last week' and 'functional limitation' as measured by the Oswestry Disability Index ODI. A negative correlation with a resource factor was found in SF12 'physical health' score and 'education status'. The strongest risk factor was 'pain intensity last week', the strongest resource 'physical health' (Table 3).

'Delayed presentation' demonstrated statistically significant correlations with 14 prognostic factors; positive correlation with risk factors included one biomedical factor ('functional limitation'), four occupational risk factors ('resigned attitude towards the job', 'uncertainty', 'problem with organisation of work tasks', 'work interruptions'), six psychological risk factors 


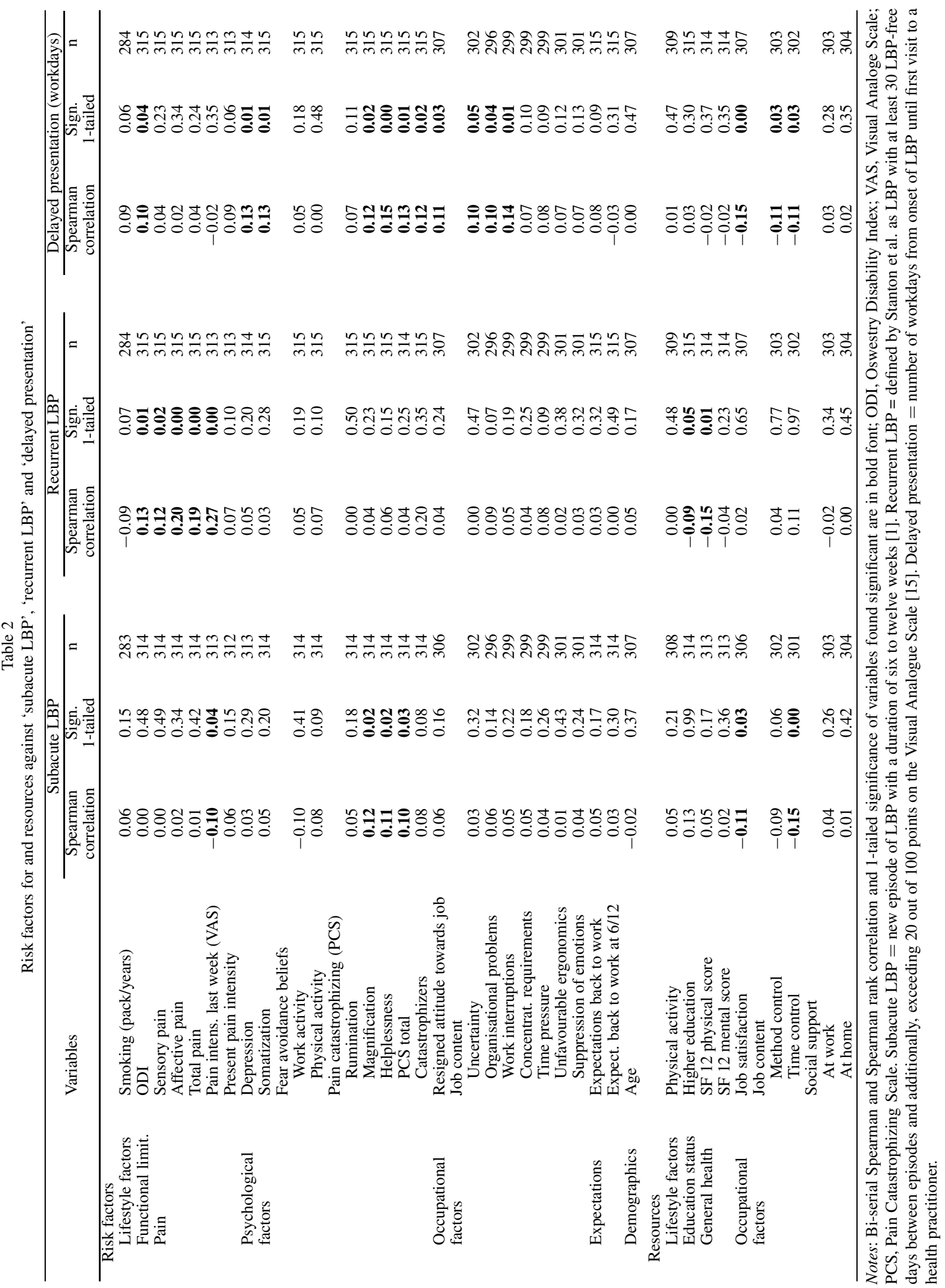


Table 3

Risk factors for and resources against LBP

\begin{tabular}{|c|c|c|}
\hline & Variables & Description (where appropriate) \\
\hline \multicolumn{3}{|l|}{ Risk factors } \\
\hline Lifestyle factors & Smoking (pack/years) & Packs of cigarettes smoked per year \\
\hline Functional limitation & ODI & Oswestry Disability Index \\
\hline \multirow[t]{5}{*}{ Pain } & Sensory pain & All items taken from McGill Pain Questionnaire \\
\hline & Affective pain & \\
\hline & Total pain & \\
\hline & Pain intensity last week & On Visual Analogue Scale (VAS) \\
\hline & Present pain intensity & \\
\hline \multirow[t]{11}{*}{ Psychological factors } & Depression & Defined by ZUNG Depression Index \\
\hline & Somatization & Defined by Modified Somatic Perception Questionnaire \\
\hline & Fear avoidance beliefs & Defined by Fear Avoidance Beliefs Questionnaire \\
\hline & Work activity & \\
\hline & Physical activity & \\
\hline & Pain catastrophizing & Defined by Pain Catastrophizing Score (PCS) \\
\hline & Rumination & To turn a matter over and over in the mind \\
\hline & Magnification & Magnifying one's actual symptoms \\
\hline & Helplessness & \\
\hline & PCS total & \\
\hline & Catastrophizers & Score on PCS of $>24$ out 52 points \\
\hline \multirow[t]{9}{*}{ Occupational factors } & Resigned attitude towards job & Working in current position due to lack of alternatives \\
\hline & Job content & \\
\hline & Uncertainty & \\
\hline & Organisational problems & \\
\hline & Work interruptions & \\
\hline & Concentration requirements & \\
\hline & Time pressure & \\
\hline & Unfavourable ergonomics & \\
\hline & Suppression of emotions & \\
\hline \multirow{2}{*}{ Expectations } & Expectations going back to work & \\
\hline & Expect. back to work at 6 months & \\
\hline Demographics & Age & \\
\hline \multicolumn{3}{|l|}{ Resources } \\
\hline Lifestyle factors & Physical activity & \\
\hline Education status & Higher education & \\
\hline \multirow[t]{2}{*}{ General health } & SF 12 physical score & Short Form-12 Questionnaire \\
\hline & SF 12 mental score & \\
\hline \multirow[t]{7}{*}{ Occupational factors } & Job satisfaction & \\
\hline & Job content & \\
\hline & Method control & E.g., independently plan and organize one's own work \\
\hline & Time control & E.g., influence on work scale and schedule \\
\hline & Social support & \\
\hline & At work & \\
\hline & At home & \\
\hline
\end{tabular}

('depression', 'somatization', 'pain catastrophizing', 'catastrophizer' status, 'magnification', 'helplessness'). Negative associations with occupational resource factors included 'job satisfaction', 'method control', and 'time control'. The strongest risk factor was 'helplessness', the strongest resource 'job satisfaction' (Table 3). Spearman rank correlations confirm the previously reported findings.

While the reported correlations so far were in line with directional expectations and turned out to be significant in one-tailed significance testing, many correlations did not. The number of significant associations and the meaningful pattern of significant correla- tions across time-related LBP characteristics, however, rule out results that simply reflect significant coefficients turned out by chance in performing many tests. The random expectation of significant correlations per time-related indictor would be $5 \%$, equalling one or two significant correlation coefficients in 36 correlation tests.

\section{Discussion}

The current study found that the presence of potential risk factors for the development persistent LBP 
correlated with all time-related variables ('subacute LBP', 'recurrent LBP', and 'delayed presentation') as hypothesised. Further, the presence of resources for persistent LBP prevention were inversely correlated with all time-related variables, suggesting that in all conditions, a lack of certain preventative resources is present. Most interestingly, the time-related variables of LBP had different patterns of risk factors for developing persistent LBP present. Participants with 'subacute LBP' on presentation and those with 'delayed presentation' displayed similar risk profiles including psychological risk factors and occupational resource factors; however, those with 'recurrent LBP' had different potential risk factors present including 'pain intensity' and 'functional limitation'. Further, different patterns of resources for preventing persistent LBP were also found between these time-related variables.

That there was a substantial overlap between the patterns of prognostic factors of the time-related variables 'subacute LBP' and 'delayed presentation' is not surprising as these two time-related variables are expected to correlate. As individuals suffering from subacute LBP are somehow adapted to their LBP they may wait for a longer time to seek primary care. For both variables occupational and psychological aspects were revealed to be essential risk factors, with occupational aspects also revealed to be essential resources (e.g. lack of occupational resources was present in this sample). In contrast, the pattern of prognostic factors for 'recurrent LBP' proved to be considerably different comprising mainly biomedical risk factors and resources.

It is unclear why 'recurrent LBP' would be associated with biomedical risk factors such as 'pain intensity' or 'physical health' status and not associated with any psychological or occupational risk factors. This suggests that the development of persistent pain in those with 'recurrent LBP' may be for different reasons than the development of persistent pain in people with 'subacute LBP' or 'delayed presentation' of LBP. Alternatively, associations with psychological and occupational risk factors may have decreased in time, i.e. in those with recurrent pain associations were stronger when the first episode appeared but decreased afterwards when other factors were more involved in susceptibility to recurrence of LBP. 'Recurrent LBP' and prolonged LBP are different entities with different risk factors and resources. Delayed care-seeking behaviour may be due to higher work loads, work stress or the expectation of self-healing.

To our knowledge this is the first investigation of correlation between LBP status at initial presentation to a primary care health practitioner and the presence of potential risk factors and preventative resources for developing persistent LBP. One strength of the current study is that we measured this LBP status interval in workdays - known to be a better indicator for a predominantly working population than a general count of days. Another strength is that $93 \%$ of the correlations and the inverse correlations - although not all significant - showed the same direction as expected.

The limitations of our study include possible undersampling of non-English speaking participants, as due to the mailed questionnaire format of data collection we were unable to provide alternatives for people that did not adequately understand English to complete the questionnaires. Also the strength of the reported correlations between 0.1 and 0.3 has to be considered as small according to the convention of Cohen. Furthermore, for the two binary time-related variables 'subacute' and 'recurrent LBP' it was theoretically possible to have a 'Yes' for both, 'subacute' and 'recurrent LBP'. This was, however, extremely rare with only six cases out of 315 . Thus, there is a zero association $(-0.05)$ between 'subacute' and 'recurrent LBP'. This zero association itself is potentially clinically interesting. Because of the small number of only six patients reporting both, 'subacute' and 'recurrent LBP', we hesitate to report any characteristics of this group as being clinically important. Association of 'delayed presentation' and 'recurrent LBP' was small (0.13) while 'delayed presentation' was meaningfully associated with 'subacute LBP' (0.49), meaning that these patients waited with their first consultation until their LBP had become 'subacute'.

Implications for clinical practice are threefold; (1) LBP patients in primary care with prolonged symptoms or delayed care-seeking behavior, who are at risk of developing persistent LBP, should be screened for psychological factors such as 'magnification' and 'helplessness'; (2) In these patients, occupational resources like 'time control' and 'job satisfaction' should be addressed and strengthened in primary and secondary prevention, where feasible; (3) Patients with recurrent LBP do not benefit from such a screening as these patients might have already adapted their work to their recurrent LBP episodes; in this patient group questions in regard to their work history, i.e. previous work conditions, might provide further insights.

Future research should examine time-related variables that influence prognostic factors for LBP in different clinical settings such as predominantly retired or unemployed populations. Furthermore, the pattern of 
delayed care-seeking behaviour in patients with 'subacute LBP' or increased 'delayed presentation' should be investigated, looking in more detail at workloads and work stress as well as patient expectations of selfhealing. As 'recurrent LBP' and prolonged LBP are different entities, their diverse association with occupational and other factors for LBP in primary care should be carefully considered when drawing conclusions about their prognosis from LBP inception cohorts. Further longitudinal studies should test whether associations are directional in time, restricted to paths from time variables to risk and resource factors or reverse or whether mutual associations in time exist.

\section{Conclusion}

In this study of patients with acute and subacute LBP from a primary care setting, risk factors for persistent LBP correlated with the three time-related variables 'subacute LBP', 'recurrent LBP' and 'delayed presentation'. Resources for preventing persistent LBP inversely correlated with these time-related variables. Further, the presence of potential risk factors for developing persistent LBP (as well as the lack of resources for preventing LBP) was different between those with 'subacute LBP'/ 'delayed presentation' and those with 'recurrent LBP'. Hence, the influence of time-related variables (e.g. 'delayed presentation' and/or the presence of 'recurrent LBP') on prognostic occupational, psychological, social and biomedical factors for acute and subacute LBP should be carefully investigated when drawing conclusions from LBP inception cohorts. Time-related LBP characteristics and especially 'delayed presentation' of first LBP to primary carers should be assessed more often and tested for their prognostic value in further studies.

\section{Acknowledgements}

We gratefully acknowledge Kirsten Stout from the Centre for Musculoskeletal Outcomes Research (CMOR) at Dunedin School of Medicine, University of Otago for developing and maintaining the documentation and data management system. Furthermore, we would like to thank Cathy Chapple from CMOR for critical comments on the draft of our manuscript and all participating patients and health practitioners for their time and effort. This work was supported by Lottery Health Research [LHR264307, LHR278283]; the
Wishbone Trust of New Zealand [reference 03/09, reference 08/10]; the Bruce McMillan Trust [reference 11/08]; the Dunedin School of Medicine [reference 08/08]; realHealth International [reference 05/08]; and the University of Berne [reference 2009]. MM was funded by a scholarship awarded by the University of Otago. TRS received funding through a Canadian Institutes of Health Research Postdoctoral Training Fellowship.

\section{References}

[1] Hilfiker R, Bachmann LM, Heitz CAM, Lorenz T, Joronen $\mathrm{H}$, Klipstein A. Value of predictive instruments to determine persisting restriction of function in patients with subacute non-specific low back pain. Systematic review. Eur Spine J. 2007;16(11):1755-75.

[2] Melloh M, Elfering A, Chapple CM, Kaser A, Rolli Salathe C, Barz T, et al. Prognostic occupational factors for persistent low back pain in primary care. Int Arch Occup Environ Health. Epub 2012 Mar 21.

[3] Katz JN. Lumbar disc disorders and low-back pain: socioeconomic factors and consequences. J Bone Joint Surg Am. 2006;88 Suppl 2:21-4.

[4] Melloh M, Elfering A, Egli Presland C, Roder C, Hendrick P, Darlow B, et al. Predicting the transition from acute to persistent low back pain. Occupational medicine. 2011;61(2):12731. Epub 2011/01/05.

[5] Lonnberg F, Pedersen PA, Siersma V. Early predictors of the long-term outcome of low back pain-results of a 22 year prospective cohort study. Fam Pract. 2010;27(6):609-14. Epub 2010/08/05.

[6] Pincus T, Santos R, Breen A, Burton AK, Underwood M. A review and proposal for a core set of factors for prospective cohorts in low back pain: a consensus statement. Arthritis Rheum. 2008;59(1):14-24. Epub 2008/01/01.

[7] Hay EM, Dunn KM. Prognosis of low back pain in primary care. BMJ. 2009;339:b3694. Epub 2009/10/08.

[8] Sackett D, Haynes R, Guyatt G, Tugwell P. Clinical Epidemiology: Little Brown \& Company; 1991.

[9] Last J. A Dictionary of Epidemiology: Oxford University Press; 2001.

[10] Henschke N, Maher CG, Refshauge KM, Herbert RD, Cumming RG, Bleasel J, et al. Prognosis in patients with recent onset low back pain in Australian primary care: Inception cohort study. BMJ. 2008;337:a171. Epub 2008/07/11.

[11] Costa Lda C, Maher CG, McAuley JH, Hancock MJ, Herbert $\mathrm{RD}$, Refshauge KM, et al. Prognosis for patients with chronic low back pain: inception cohort study. BMJ. 2009;339:b3829. Epub 2009/10/08.

[12] Elfering A. Work-related outcome assessment instruments. Eur Spine J. 2006;15 Suppl 1:S32-43.

[13] Aghayev E, Sprott H, Bohler D, Roder C, Muller U. Sleep quality, the neglected outcome variable in clinical studies focusing on locomotor system; a construct validation study. BMC Musculoskelet Disord. 2010;11:224.

[14] Melloh M, Aebli N, Elfering A, Roder C, Zweig T, Barz T, et al. Development of a screening tool predicting the transition from acute to chronic low back pain for patients in a GP setting: Protocol of a multinational prospective cohort study. BMC Musculoskelet Disord. 2008;9:167. Epub 2008/12/23. 
[15] Stanton TR, Latimer J, Maher CG, Hancock MJ. A modified Delphi approach to standardize low back pain recurrence terminology. Eur Spine J. 2011;20(5):744-52.

[16] Balague F, Mannion AF, Pellise F, Cedraschi C. Clinical update: low back pain. Lancet. 2007;369(9563):726-8.

[17] Airaksinen O, Brox JI, Cedraschi C, Hildebrandt J, KlaberMoffett J, Kovacs F, et al. Chapter 4. European guidelines for the management of chronic nonspecific low back pain. Eur Spine J. 2006;15 Suppl 2:S192-300.
[18] van Tulder M, Becker A, Bekkering T, Breen A, del Real MT, Hutchinson A, et al. Chapter 3. European guidelines for the management of acute nonspecific low back pain in primary care. Eur Spine J. 2006;15 Suppl 2:S169-91.

[19] Statistics NZ. Census. Wellington: 2006.

[20] Statistics NZ. Household Labour Force Survey. Wellington: 2011.

[21] Wellington: New Zealand Statistics; [cited 2011 6th April]; Available from: www.stats.govt.nz. 